\title{
Evaluating Residual Errors in Waveguide Network Analysers from Microwave to Submillimetre-wave Frequencies
}

\author{
L Stant $^{1,2,3}$, P H Aaen ${ }^{1,2}$ and N M Ridler ${ }^{1,3}$ \\ ${ }^{1}$ Nonlinear Microwave Measurement \& Modelling Laboratories, University of Surrey \& National Physical \\ Laboratory, UK \\ ${ }^{2}$ Advanced Technology Institute, University of Surrey, Guildford, GU2 7XH, UK \\ ${ }^{3}$ Time, Quantum and Electromagnetics Division, National Physical Laboratory, Teddington, TW11 0LW, UK
}

\begin{abstract}
Vector network analysers (VNA) are used extensively for measurements that are made at frequencies ranging from a few kilohertz to at least one terahertz. At radio and microwave frequencies, there are well-established methods for assessing the quality and confidence of these measurements, when they are made in coaxial lines. These methods are usually based on determining the size of residual errors that remain in the VNA after calibration. To date, the performance of these methods has not been investigated in rectangular waveguide, and, at millimetre- and submillimetre-wave frequencies. This paper investigates the application of one of these techniques for waveguide measurements at microwave, millimetre- and submillimetre-wave frequencies. Typical values of residual errors obtained over these frequency ranges are given. These values are considered representative and so can be used by other users of waveguide VNAs to compare with values obtained on their own systems, therefore helping to verify the performance of their systems.
\end{abstract}

\section{Introduction}

For many years (dating back at least 50 years [1]), the Vector Network Analyser (VNA) has been a major measurement technology for many applications that use high-frequency electromagnetic signals. The early versions of VNAs operated mainly at radio-frequency and microwave frequencies (i.e. from a few kilohertz to a few tens of gigahertz) [2,3]. These systems rapidly developed and matured, and soon were being used for many diverse measurement applications. Many of these applications required both accurate and reliable measurements from these VNAs. This is particularly so in areas such as manufacturing, calibration and testing. This is often driven by requirements given in international Quality Management documents such as the ISO 9000 series of standards [4] (for manufacturing and process control) and the ISO 17025 standard [5] (for calibration and testing).

The requirements given in these international standards are for measurements that can be demonstrated as fitfor-purpose (in terms of the achievable level of accuracy, etc) and made traceable to the international system of units $[6,7]$. These requirements are not trivial for a VNA due to the complicated nature of the VNA's operating principles. This led to much work by experts to develop methods that addressed these needs in ways that were suitable for use by end-users in the manufacturing, calibration and testing communities. Much of this work was undertaken by the ANAMET Technology Group (www.npl.co.uk/anamet) during the 1990s. This resulted in a series of reports [8-10] describing the development of a guidance document that gave a procedure for assessing the performance of calibrated VNAs. The resulting guidance document [11] was published by the European cooperation for Accreditation (EA, www.european-accreditation.org) so that laboratories operating to the ISO 17025 standard and/or ISO 9000 series of standards could implement the method for their own purposes. Ownership of this EA document was later transferred to the European Association of National Metrology Institutes (EURAMET) and re-published [12] as part of their Calibration Guides series of documents. This is now the current version of this document and is available as a free download from the EURAMET web-site (http://www.euramet.org).

Another activity that was taking place around the same time as the development of the guidance documents, was work by the VNA manufacturers to extend the operating frequency range. Several techniques were developed to extend the frequency range into the millimetre-wave region (i.e. from $30 \mathrm{GHz}$ to $300 \mathrm{GHz}$ ). These systems (see, for example, [13-15]) often relied on using rectangular metallic waveguide for the VNA test ports to propagate the test signals at these frequencies. In more recent years, the upper operating frequency for these 
waveguide-based VNAs has been further extended, into the submillimetre-wave region (see, for example, [16-18]). VNAs are now available that operate at frequencies to at least $1.1 \mathrm{THz}$ [19].

The EURAMET Calibration Guide [12] is only intended for VNAs operating at microwave frequencies (i.e. to approximately $30 \mathrm{GHz}$ ). The Guide does not address VNAs operating at millimetre-wave and submillimetrewave frequencies. In addition, the EURAMET Guide only deals with VNAs that make measurements using coaxial connectors. The Guide does not include VNAs that make measurements in waveguide. There are no international guidelines available for VNA users making measurements in waveguide, either at microwave or millimetre- and submillimetre-wave frequencies. Kishikawa et al [20] have presented an evaluation of VNA performance in waveguide at millimetre- and submillimetre-wave (up to $330 \mathrm{GHz}$ ) frequencies using several techniques, but no investigation into the validity of such techniques when used in waveguide has been published.

This paper investigates a method for verifying VNA measurements in waveguide at frequencies up to $750 \mathrm{GHz}$. The method is an adaptation of the method described in the EURAMET Guide [12] for verifying VNA measurements made in coaxial lines at microwave frequencies. The paper begins by describing the method given in the EURAMET Guide and showing how it can be adapted for making measurements in rectangular waveguide at microwave frequencies. The performance achieved using the technique in waveguide is compared with equivalent measurements (i.e., over a similar frequency range) made in coaxial lines. The paper then, for the first time, examines the applicability of the technique for VNA measurements made in waveguide operating at millimetre- and submillimetre-wave frequencies. A discussion is included into effects caused by mismatches, occurring at the VNA measurement reference planes, when measuring in waveguide with small aperture dimensions (i.e., as used at millimetre- and submillimetre-wave frequencies). Finally, recommendations are given concerning using the technique by end-users wishing to verify their own waveguide VNA measurements at microwave, millimetre-wave and submillimetre-wave frequencies.

\section{Method}

The EURAMET Guide [12] presents a process for evaluating the uncertainty of measurements performed on a calibrated VNA, allowing users to verify that values measured using the instrument are of acceptable accuracy. This process involves measuring a selection of dominant contributions to measurement uncertainty and combining them appropriately. Contributions include both systematic errors, which remain constant over the period of measurements, and random errors, which do not. The error model for voltage reflection coefficient $(\Gamma)$ measurements performed with a VNA is represented by the following equations for one-port (1) and two-port (2) measurements [12]:

$$
\begin{gathered}
U_{\Gamma}=D+T \Gamma+M \Gamma^{2}+R_{\Gamma} \\
U_{\Gamma}=D+T \Gamma+M \Gamma^{2}+R_{\Gamma}+S_{21}^{2} \Gamma_{L}
\end{gathered}
$$

where $U_{\Gamma}$ is the combined uncertainty in the measurement, $D$ is the residual directivity, $T$ is the effect of tracking and nonlinearity, $M$ is the residual test port match (TPM), $\Gamma$ is the measured voltage reflection coefficient, $R_{\Gamma}$ represents all the random contributions, $S_{2 l}$ is the measured transmission coefficient of the device-under-test (DUT), and $\Gamma_{\mathrm{L}}$ is the residual load match. The most significant systematic error contributors to the measurement uncertainty are, in most cases, the directivity and TPM.

In order to measure $\Gamma$, the VNA must separate reflected and incident voltage waves and then sample them using complex receivers. However, various components in the signal path may cause a portion of the incident wave to leak into the reflected wave receiver without having reached the DUT. This directivity error should be removed by applying correction terms extracted during the VNA calibration. However, as no calibration will be perfect, some residual directivity error will remain (referred to as effective directivity in [12]). To measure the residual directivity, a matched load can be connected to the test port being assessed. This should theoretically reflect none of the incident wave and the only voltage present at the reflected wave receiver should be due to the residual directivity. In practice, the match of the load will never be perfect, so it is likely that using this method the residual directivity will typically be either over- or underestimated. An improved method, used in [12] and widely accepted for use with coaxial measurements, is called the "ripple extraction technique". This uses a similar principle to measure the residual directivity, but significantly improves the accuracy of the residual error evaluation. An illustration of its method is provided in Fig. 1. 
a)

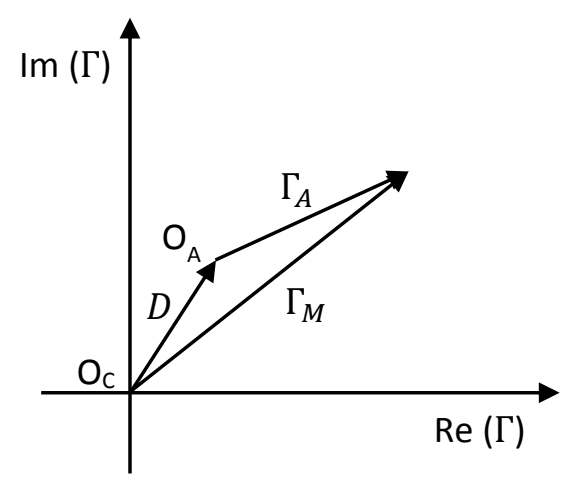

b)
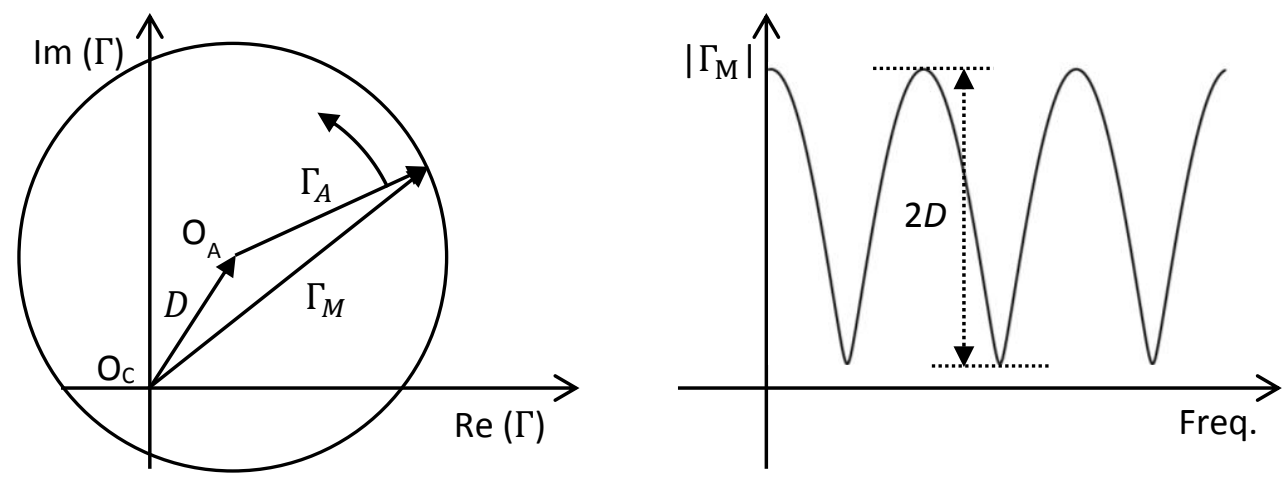

c)
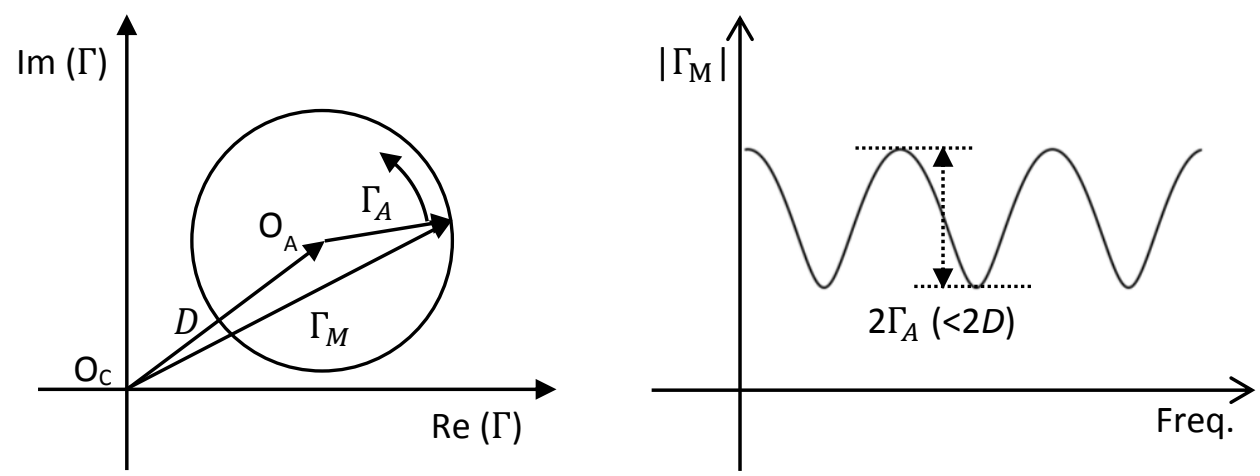

Figure 1. a) When measured on the calibrated VNA, a perfect matched load would reveal the actual origin $\left(O_{A}\right)$ on a polar plot of $\Gamma$ as offset from the calibrated origin $\left(O_{C}\right)$ by the residual directivity $(D)$. If a realistic nearmatched load offset by a line section is instead measured, $\Gamma$ as measured by the VNA $\left(\Gamma_{M}\right)$ will be the sum of the residual directivity $(D)$ and the actual $\Gamma\left(\Gamma_{A}\right)$. b) As $\Gamma_{M}$ is measured across a swept frequency range, the phase change in the line increases causing the phase of $\Gamma_{A}$ to sweep also. This rotates $\Gamma_{A}$, resulting in ripples in the plot of $\left|\Gamma_{M}\right|$ against frequency. The magnitude of the ripples is equal to $2 D$. c) However, if $\Gamma_{A}<D$, then the ripple magnitude is now $2 \Gamma_{A}$ instead of $2 D$ and the residual directivity as evaluated using the ripple extraction technique would be underestimated.

To perform the ripple extraction technique, a short length of line is connected to the test port, to the end of which is added a near-matched load covering the frequency range under test. The critical dimensions of the line section (length and radii) should be traceable to national standards and have a characteristic impedance identical to that of the VNA setup. For these reasons a beadless coaxial airline is suggested [12]. The load can be either the same as used for calibration or another with $0.1 \leq|\Gamma| \leq 0.2$ (in linear units) to ensure that $|\Gamma| \geq|D|[12]$. If $|\Gamma|<|D|$, then the measured residual directivity will be underestimated as explained by Fig. 1c. If the calibration matched load is used for the measurement, the small reflection from a second connection and any loss in the 
airline will cause $\Gamma$ to be greater than the residual directivity from the original measurement of the load. Alternatively, because $|\Gamma|<0.1$ for the matched load used for calibration, using another load with a known higher $|\Gamma|$ ensures that there is no underestimate. Once the instrument has been configured, $\Gamma$ is measured and the magnitude plotted against frequency using a linear scale. A ripple will then be visible on the trace, from which the residual directivity can be calculated from the maximum ripple amplitude (MRA):

$$
D=\frac{M R A_{\text {Matched-Load }}}{2}
$$

For coaxial measurements as specified in [12], there is a high probability that the condition required to avoid underestimation of $|D|$ is met. However, in order to assess the suitability of the technique in waveguide a method of assessing this condition has been used. By examining either a complex plot (polar or Smith chart) or a phase plot, the geometric symptom shown in Fig. 1c can be identified. When using a complex plot, the origin should lie within the circumference of the reflection coefficient trace for a valid determination of the residual error to be achieved. For any frequency range where it does not, the ripple technique provides an underestimation of the residual error. When using a phase plot, there will be regular wrapping of the reflection coefficient phase for frequency ranges where the residual error is correctly measured, whereas when underestimation occurs the phase will vary by less than 180 degrees per period. An example of both plots are shown in Fig. 2. Either of these methods can be used to identify when a calibration and the ripple extraction technique needs to be repeated. If the repeat measurements still fail the test, then the choice of loads may need to be altered.

a)

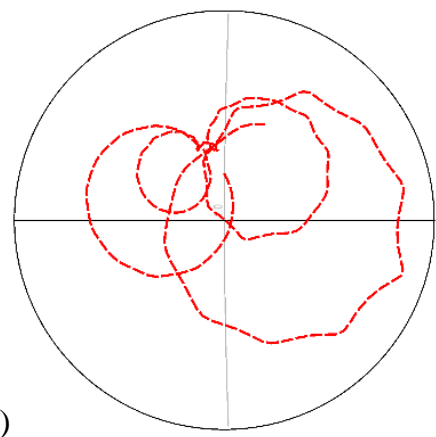

b)
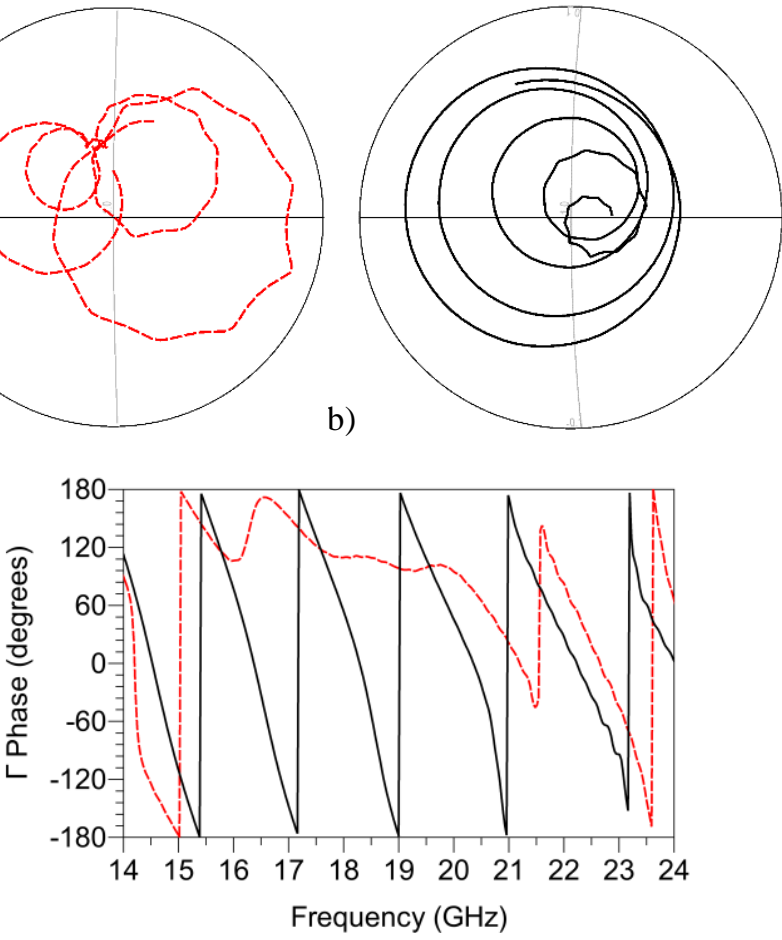

Figure 2. Measurements of residual directivity plotted over the frequency range of $14 \mathrm{GHz}$ to $24 \mathrm{GHz}$ performed in coaxial transmission line using both the calibration load and a load from a different calibration kit. a) A Smith chart representation, magnified about the origin, of the calibration load result. b) A similar Smith chart representation of the different load result. The intersection of the two grid lines on the Smith charts represents the origin of the plot $(\mid \Gamma=0)$, and both are scaled separately for clarity. $c)$ The phase plot of both results, with the calibration load represented by the red dotted trace and the different load by the black solid trace. The result from the different load indicates an accurate residual directivity estimate across the entire measured spectrum. The result from the calibration load shows an underestimate of the residual directivity is likely between $16 \mathrm{GHz}$ and $22 \mathrm{GHz}$.

TPM is caused by imperfections in the impedance match between components in the VNA setup. This causes reflections that interfere with the DUT measurement and therefore introduce error. Calibration also corrects for TPM, but as with directivity some residual error will remain. To measure residual TPM, a short-circuit can be 
connected to the test port being assessed. This should reflect the entire incident signal and maximise reflections in the VNA setup. If significant residual TPM error is present, then the measured $\Gamma$ will be less than 1 . However, the short-circuit may not provide a perfect reflection and so the ripple extraction technique is favoured for this measurement also.

To measure residual TPM using the ripple extraction technique, the same procedure as for residual directivity is followed but the matched load at the end of the line is replaced by a short-circuit. A similar plot is acquired and the residual TPM, $M$, is given by:

$$
M=\frac{M R A_{\text {Short-Circuit }}}{2}
$$

Because the reflection coefficient for this measurement should be close to 1 , there is no risk that this value will be greater than the true residual TPM and cause an underestimate as has been shown to be the case for residual directivity. This is shown in Fig. 3, where the origin of the Smith chart is clearly inside the circular trace (Fig. 3a) and the phase consistently wraps across the measured bandwidth (Fig. 3b).
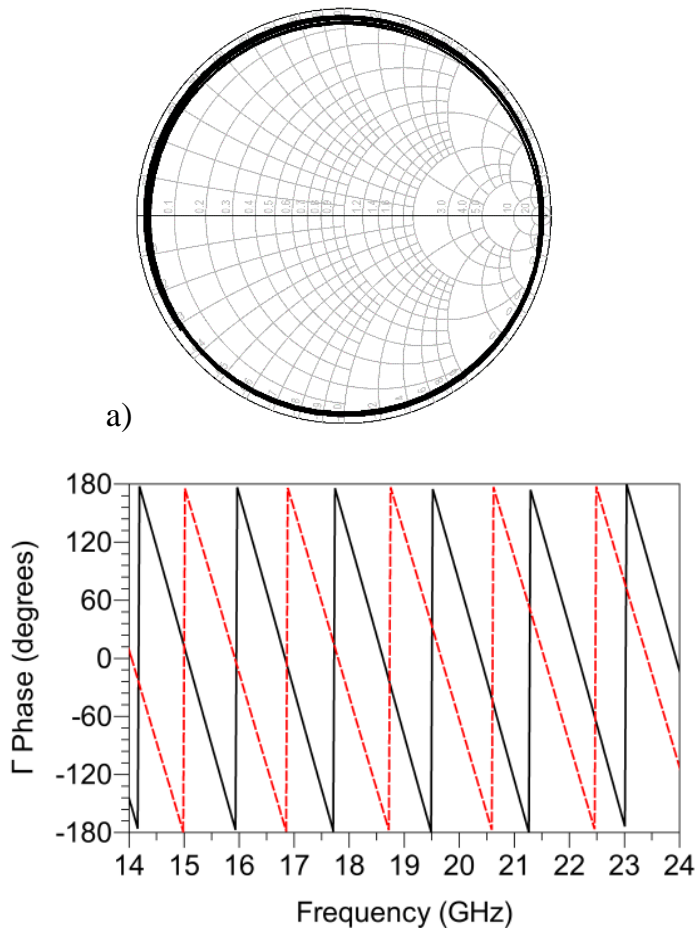

Figure 3. Measurements of residual TPM plotted over the frequency range of $14 \mathrm{GHz}$ to $24 \mathrm{GHz}$ performed in coaxial transmission line. a) The Smith chart representation of the result when using the calibration shortcircuit. The result when using a short-circuit from a different calibration kit appears almost identical. b) The phase plot of the results when using the calibration short-circuit (dotted trace) and the different short-circuit (solid trace). Both measurements indicate a valid residual TPM estimate across the entire measured bandwidth. Residual TPM measurements avoid the risk of underestimation via the mechanism described for residual directivity measurements due to $\Gamma$ being close to unity (i.e., far from the origin of the Smith chart).

To perform the ripple extraction technique for both described residual error sources requires just three components: A short-circuit, a near-matched load, and a short section of line. These components are realizable in both coaxial and rectangular waveguide, so the technique is physically possible to perform in waveguide setups. In the following investigation the residual systematic errors from two calibrations, three-known-loads and through-reflect-line (TRL), will be determined using the ripple extraction technique described in this paper. In coaxial line, the short-open-load-thru (SOLT) variant of the former is used. However, in waveguide an opencircuit is not straightforward to realise or widely adopted, so a common variant of SOLT calibration which uses an offset short (SOSLT) will be used instead. 


\section{Microwave frequencies}

\subsection{Coaxial Line}

The ripple extraction technique was performed in coaxial line in accordance with the EURAMET Guide [12] instruction as described in Section 2. The guide provides a range of typical values for both residual directivity and TPM ripple measurements with which our results can be compared with. All measurements presented in this paper were acquired using a Keysight 5247A PNA-X VNA fitted with $1.85 \mathrm{~mm}$ test ports attached to flexible port extender cables with ruggedised connectors. The coaxial measurement setup used a $75 \mathrm{~mm}$ beadless airline and the calibration kit matched load and short-circuit, connected as described in Section 2. Fig. 4 shows the ripple trace obtained by plotting $|\Gamma|$ against frequency for a residual directivity measurement on both ports using a SOLT calibration. Fig. 5 shows a similar ripple trace obtained with a short-circuit instead of a matched load, which is caused by the residual TPM. The results of the measurements, along with the expected ranges provided in [12], are presented in Table 1. It can be seen that the measured values for the coaxial line setup fall within the typical ranges specified by [12].

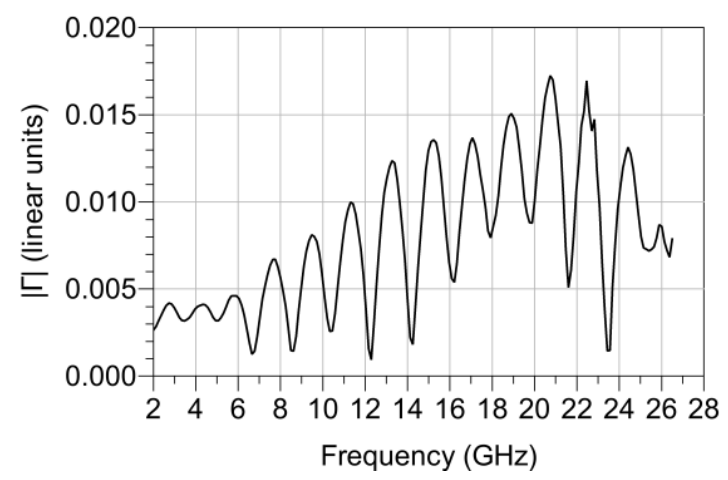

Figure 4: Magnitude of the reflection measurement of a matched load offset by a short line length in coaxial line. The VNA was calibrated using the SOLT method. Apart from the dominant ripple, other variations in $|\Gamma|$ are due to the imperfect response of the matched load and the beadless line.

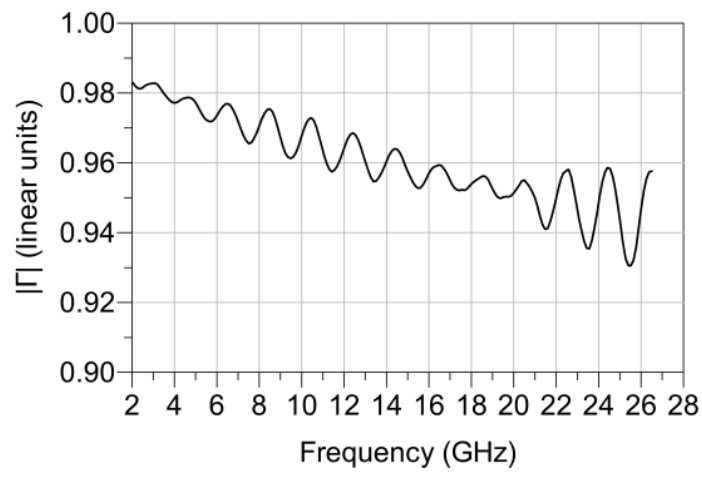

Figure 5: Magnitude of the reflection measurement of a short circuit offset by a short line length in coaxial line. The VNA was calibrated using the SOLT method, and measurements were performed on both port 1 (solid line) and port 2 (dotted line). The increasing attenuation with frequency is characteristic of small losses in the beadless airline.

Table 1: Residual directivity and TPM values obtained for $3.5 \mathrm{~mm}$ coaxial line VNA calibrations as measured by the ripple extraction method. Both SOLT and TRL calibration techniques were assessed. The range of representative residual error values from [12] has also been included for comparison.

\begin{tabular}{ccccc}
\hline \multirow{2}{*}{ Cal. Type } & \multicolumn{2}{c}{ Residual Directivity } & \multicolumn{2}{c}{ Residual TPM } \\
\cline { 2 - 5 } & Port 1 & Port 2 & Port 1 & Port 2 \\
\hline SOLT & 0.008 & 0.009 & 0.014 & 0.010 \\
TRL & 0.008 & 0.007 & 0.002 & 0.002 \\
EURAMET Guide [12] & $0.002-0.02$ & $0.002-0.02$ & $0.005-0.02$ & $0.005-0.02$ \\
\hline
\end{tabular}




\subsection{Waveguide}

The same method was applied to two different sizes of centimetre band rectangular metallic waveguide, WR-90 and WR-42. These waveguides have usable frequency ranges of $8.2 \mathrm{GHz}$ to $12.4 \mathrm{GHz}$ and $18.0 \mathrm{GHz}$ to $26.5 \mathrm{GHz}$ respectively. In order to avoid the effects of non-propagating (evanescent) modes created by the waveguide to coaxial adapter, an appropriate length of straight waveguide was attached to each adapter where possible and the measurement planes defined at the end of the lines. The results of the ripple extraction technique for the two waveguide sizes are shown in Table 2:

Table 2: Residual directivity and TPM values of WR-90 and WR-42 waveguide VNA calibrations as measured by the ripple extraction method. Both SOSLT and TRL techniques were used to calibrate the VNA.

\begin{tabular}{ccccccc}
\hline \multirow{2}{*}{$\begin{array}{c}\text { Freq. } \\
(\mathrm{GHz})\end{array}$} & Waveguide & Cal. Type & \multicolumn{2}{c}{ Residual Directivity } & \multicolumn{2}{c}{ Residual TPM } \\
\cline { 4 - 6 } & Size & & Port 1 & Port 2 & Port 1 & Port 2 \\
\hline $8.2-12.4$ & WR-90 & SOSLT & 0.005 & 0.004 & 0.007 & 0.006 \\
& & TRL & 0.003 & 0.006 & 0.002 & 0.002 \\
$18-26.5$ & \multirow{2}{*}{ WR-42 } & SOSLT & 0.003 & 0.004 & 0.010 & 0.005 \\
& & TRL & 0.002 & 0.002 & 0.002 & 0.001 \\
\hline
\end{tabular}

\section{Millimetre-wave frequencies}

To perform measurements at frequencies above $50 \mathrm{GHz}$, a range of external frequency extender heads were attached to the VNA. These extender heads included a line section attached to each test port of suitable length to avoid effects caused by evanescent modes that may exist close to the test ports. To study the performance of the ripple extraction technique at millimetre wavelengths, WR-15 and WR-05 waveguides were chosen. These waveguides have operating frequency ranges of $50 \mathrm{GHz}$ to $75 \mathrm{GHz}$ and $140 \mathrm{GHz}$ to $220 \mathrm{GHz}$ respectively. The results obtained from using the ripple extraction technique are shown in Table 3:

Table 3: Residual directivity and TPM values of WR-15 and WR-05 waveguide VNA calibrations as measured by the ripple extraction method. Both SOSLT and TRL techniques were used to calibrate the VNA.

\begin{tabular}{ccccccc}
\hline \multirow{2}{*}{$\begin{array}{c}\text { Freq. } \\
(\mathrm{GHz})\end{array}$} & Waveguide & Cal. Type & \multicolumn{2}{c}{ Residual Directivity } & \multicolumn{2}{c}{ Residual TPM } \\
\cline { 4 - 6 } & Size & & Port 1 & Port 2 & Port 1 & Port 2 \\
\hline $50-75$ & WR-15 & SOSLT & 0.002 & 0.002 & 0.018 & 0.017 \\
& & TRL & 0.002 & 0.002 & 0.009 & 0.007 \\
$140-220$ & \multirow{2}{*}{ WR-05 } & SOSLT & 0.008 & 0.009 & 0.019 & 0.024 \\
& & TRL & 0.007 & 0.008 & 0.021 & 0.015 \\
\hline
\end{tabular}

\section{Submillimetre-wave frequencies}

The final stage of the investigation studied the ripple extraction technique when applied to sub-millimetre wavelength VNA setups. The waveguide size chosen for these measurements was in the $500 \mathrm{GHz}$ to $750 \mathrm{GHz}$ band (WR-1.5) for which only one frequency extender head was available. Because of the requirement for a through standard when using the TRL calibration method, only a three-known-loads calibration was performed, which was the one-port version of SOSLT (SOSL). The line section used approximately $2.54 \mathrm{~mm}$ in length and was part of a calibration and verification kit manufactured by Virginia Diodes, Inc. Fig. 6 shows the setup used during the measurement of residual directivity, with a line and matched load connected to the frequency extender head.

The results from the ripple measurements for this waveguide band are presented in Table 4. It can be seen that the residual directivity is significantly smaller than the residual TPM. By assessing the phase wrapping of the ripple trace obtained for evaluating residual directivity, shown in Fig. 7, the ripple extraction technique appears to be subject to the failure mechanism described in Section 2 and illustrated in Fig. 2c. The lack of phase wrapping across the entire operating bandwidth shows that the ripple extraction technique was not operating within the required assumptions necessary for the technique to be valid, and therefore provided an underestimate of the residual directivity. The calibration kit used for this experiment included two full sets of standards, so the matched load was swapped and the ripple extraction technique was repeated. The issue was not resolved by this 
change, so the calibration was repeated, this time using the other matched-load. The ripple extraction technique was then performed with the matched-load used for the original calibration. However, no combination of these components provided a valid residual directivity value as assessed by the phase wrapping method. A likely cause of this effect is the poor connection repeatability inherent in this waveguide size, using typical precision UG-387 flanges. If the waveguide apertures have a greater misalignment during calibration than when the ripple extraction technique is performed, the effect of the discontinuities can cause the calibration matched-load to appear to have a higher $|\Gamma|$ than the one used for the ripple extraction technique (even if the opposite were in fact true). These conditions cause the residual directivity to be underestimated as explained in Section 2.

The suggestion that poor connection repeatability affected our results is supported by the large variation in the residual TPM value. This is not subject to the same failure mechanism as described for residual directivity, so the values included in Table 4 should be accurate estimates of the residual TPM for the two calibrations shown.

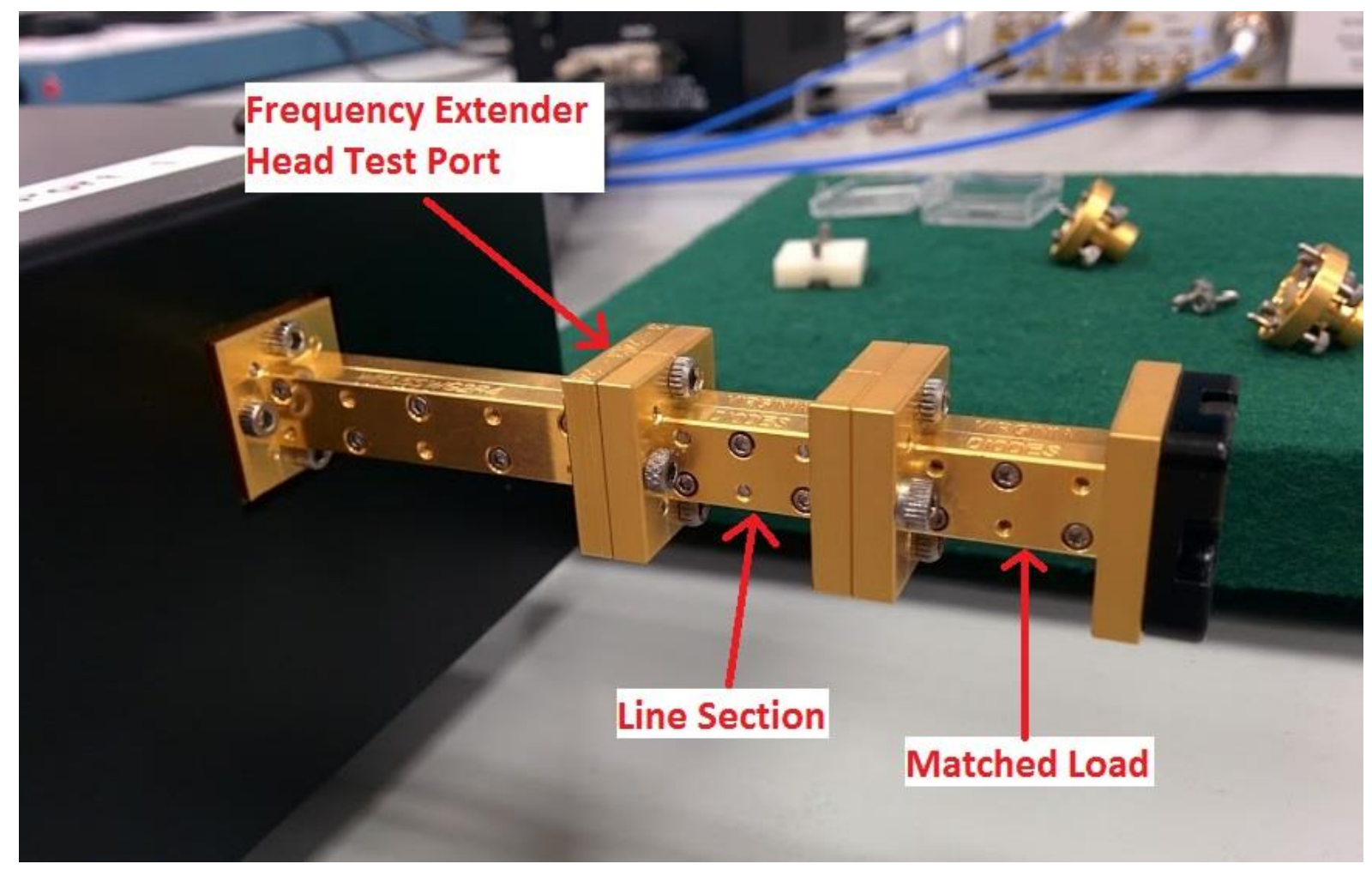

Figure 6: The test port setup used for residual directivity measurements in WR-1.5 waveguide.

Table 4: Residual directivity and TPM values of two WR-1.5 waveguide VNA calibrations as measured by the ripple extraction method. Two similar types of calibration were performed using different standards from the same kit.

\begin{tabular}{ccccc}
\hline Freq. $(\mathrm{GHz})$ & Waveguide Size & Cal. Type & Residual Directivity & Residual TPM \\
\hline $500-750$ & WR-1.5 & SOSLT Cal. 1 & 0.021 & 0.142 \\
& & SOSLT Cal. 2 & 0.025 & 0.065 \\
\hline
\end{tabular}




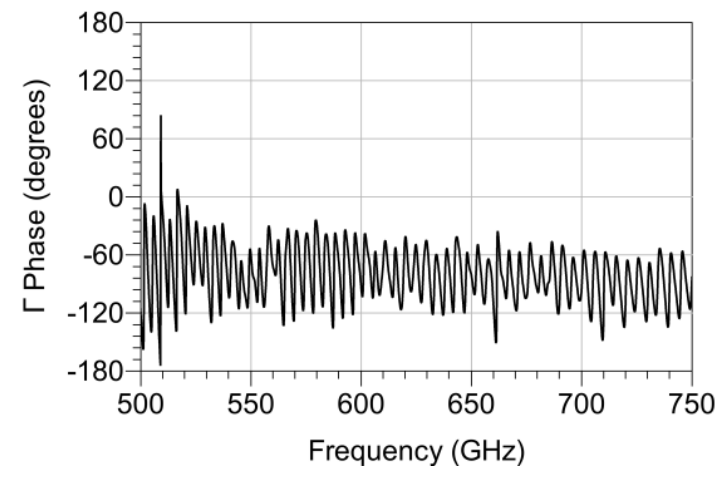

Figure 7: Phase plot for a residual directivity evaluation performed in WR-1.5 waveguide using an SOSLT calibration. The lack of phase wrapping over the entire operating bandwidth proves that in this case the ripple extraction technique will underestimate the residual error.

\section{Waveguide discontinuities}

When a discontinuity is present between two sections of rectangular waveguide, a significant reflection will be generated at the location where the two waveguides are joined together. There are several types of discontinuity possible in rectangular waveguide: E-plane and $\mathrm{H}$-plane lateral displacements, angular displacement, and corner rounding. A report produced by Bannister et al [21] presented the effects of these discontinuities at centimetre and millimetre wavelength. Subsequent work by Kerr extended this using simulations [22, 23].

The error in $\Gamma$ contributed to by the effect pf such discontinuities is proportional to wavelength and therefore also the aperture size of the waveguide. At submillimetre wavelengths, the error has been shown to be considerable [24, 25]. Recently, efforts have been made to improve the connection repeatability for waveguide at these wavelengths, and a new IEEE draft standard [26] presents three new waveguide interface types which significantly improves the alignment.

\section{Discussion}

In centimetre-wave waveguides (WR-90, WR-42), the ripple extraction technique provided values of residual directivity and TPM similar to those obtained in coaxial line. This is not surprising as providing a good quality match is used for calibration and the connections are well made and aligned, both errors should not have significant contributions outside of the VNA itself. When extended to millimetre-wave waveguide, the residual errors were found to be larger but still within the recommended values for coaxial lines. At sub-millimetre wavelengths the two calibrations that were performed resulted in significant differences in the residual errors. A likely cause for these differences is the effect of discontinuities in the waveguide components (due to poor connection repeatability and demanding mechanical tolerances) used during the calibration, especially the matched load and offset short. Additionally, by studying the phase of the measured $\Gamma$, it was shown that the ripple extraction technique was not operating within required assumptions necessary for the technique to be valid and was therefore underestimating the true value of the residual directivity. The cause of this may also be related to the poor repeatability of the waveguide connection, causing the $\Gamma$ of the matched load used for the ripple extraction to be lower than that used for the calibration. Some efforts were made to resolve this issue but were unsuccessful. New improvements to sub-millimetre wave waveguide flanges could significantly reduce this problem and allow the ripple technique to work more consistently with these very small waveguides.

A useful assessment method to test the validity of the ripple extraction technique was presented earlier in this paper, and should be performed whenever the technique is used. This assessment method views $\Gamma$ measured during the ripple extraction technique on either a phase plot, a polar plot or a Smith chart. When using a phase plot, this assessment has passed if the phase is seen to be wrapping across the operating bandwidth. When using a polar plot or Smith chart, this assessment has passed if the origin of the chart lies within the circumference of the trace. If the technique is deemed to have failed based on this assessment, the VNA should be recalibrated and the ripple extraction technique should be repeated. If this does not affect the assessment result, then the near-matched load or short-circuit used during the technique should be swapped with another (preferably known to have a higher $\Gamma$ ) and the ripple extraction technique repeated again. Only when this assessment has passed can the results from the ripple extraction technique be considered reliable. 


\section{Conclusion}

This paper investigated the effectiveness of the ripple extraction technique when applied to rectangular waveguide measurements at centimetre, millimetre and submillimetre wavelengths. Typical values of residual directivity and test port match in these three ranges have been provided. For centimetre- and millimetre-wave waveguides the ripple extraction technique works as expected and the values of residual error given in this paper can be considered representative and are suitable for users to compare their own values against. However, this paper has also shown that the ripple extraction technique may not currently be a reliable way of measuring residual error in sub-millimetre wavelength systems. The effect of discontinuities at submillimetre-wave waveguide interconnections has been considered as a cause of this issue.

\section{Acknowledgement}

The authors wish to thank Martin Salter at NPL for his assistance and advice with regards to the use of the VNA frequency extender heads. This work was part-funded through the European Metrology Research Programme (EMRP) Project SIB62, 'HF-Circuits'. The EMRP is jointly funded by the EMRP participating countries within EURAMET and the European Union.

\section{References}

[1] D Rytting, “ARFTG 50 Year Network Analyzer History”, Proc. 71 ${ }^{\text {st }}$ ARFTG Microwave Measurement Conf., pp 11-18, Atlanta, GA, USA, June 2008.

[2] R A Hackborn, “An automatic network analyser system”, Microwave J, Vol 11, No 5, pp 45-52, May 1968.

[3] S F Adam, "A new precision automatic microwave measurement system", IEEE Trans Instrumentation \& Measurement, Vol 17, No 4, pp 308-313, December 1968.

[4] "ISO 9000: International Standards for Quality Management", 4th edition, International Organization for Standardization, Genève, Switzerland, 2015. Available: www.iso.org.

[5] "ISO 17025: General Requirements for the Competence of Testing and Calibration Laboratories", 2nd edition, International Organization for Standardization, Genève, Switzerland, 2005. Available:

www.iso.org.

[6] "The International System of Units (SI)", 8th edition, International Bureau of Weights and Measures (BIPM), 2006. Available: www.bipm.org.

[7] "A concise summary of the International System of Units, the SI", International Bureau of Weights and Measures (BIPM). Available: www.bipm.org.

[8] R N Clarke, J P Ide, G R Orford and N M Ridler, "Draft EAL Procedure for the Assessment of Vector Network Analysers (VNA), ANAMET Report 002, Sept. 1996. Available from the National Physical Laboratory, Teddington, UK.

[9] J P M de Vreede, "Draft procedure for the assessment of vector network analysers (VNA)", ANAMET Report 019, Oct. 1998. Available from the National Physical Laboratory, Teddington, UK.

[10] J P M de Vreede, "Draft EA Guidance Document: Assessment of Calibrated Vector Network Analysers (VNA)", ANAMET Report 026, Aug. 1999. Available from the National Physical Laboratory, Teddington, UK.

[11] "EA Guidelines on the Evaluation of Vector Network Analysers (VNA)", European co-operation for Accreditation, Publication Reference EA-10/12, May 2000.

[12] "Guidelines on the Evaluation of Vector Network Analysers (VNA)", EURAMET cg-12, Calibration Guide, Version 2.0, 03/2011.

[13] J Cauffield and R Pollard, "A High Performance Millimeter Wave Vector Network Analyzer System", Proc. 24 ${ }^{\text {th }}$ ARFTG Microwave Measurement Conf., pp 105-118, Columbia, MD, USA, Dec. 1984. 
[14] J Fitzpatrick, "Survey of Millimeter-wave Network Analyzers", Proc. 23 ${ }^{\text {rd }}$ ARFTG Microwave Measurement Conf., pp 107-119, Santa Rosa, CA, USA, June 1984.

[15] C Oleson and A Denning, "Millimeter Wave Vector Analysis Calibration and Measurement Problems Caused by Common Waveguide Irregularities", Proc. 56 ${ }^{\text {th }}$ ARFTG Microwave Measurement Conf. Boulder, CO, USA, December 2000.

[16] OML (Oleson Microwave Labs), "Frequency extension source modules to extend signal capability from 50 to 325 GHz", Microwave Journal, vol. 47, no. 3, pp. 124-134, Mar. 2004.

[17] C Oleson, A Denning, and Y Lau, “325 to $500 \mathrm{GHz}$ Vector Network Analysis System”, in Proc. 66th ARFTG Microwave Measurement Conf., pp. 16-22, Washington, DC, USA, Dec. 2005.

[18] H Li, A Arsenovic, J L Hesler, A R Kerr, and R M Weikle, II, "Repeatability and Mismatch of Waveguide Flanges in the 500-750 GHz Band", IEEE Trans. Terahertz Science and Technology, vol. 4, no. 1, pp. 39-48, Jan. 2014.

[19] T W Crowe, B Foley, S Durant, K Hui, Y Duan, and J L Hesler "VNA frequency extenders to 1.1 THz", in 36th International Conference on Infrared, Millimeter and Terahertz Waves (IRMMW-THz), Houston, TX, USA, Oct. 2011.

[20] R. Kishikawa, M. Horibe and M. Shida, "Evaluation of complex residual error in vector network analyzer measurement system in the range of millimeter-wave and submillimeter-wave frequencies," Microwave Measurement Conference (ARFTG), 2011 77th ARFTG, Baltimore, MD, 2011, pp. 1-7.

[21] D J Bannister, E J Griffin, and T E Hodgetts, “On the Dimensional Tolerances of Rectangular Waveguide for Reflectometry at Millimetric Wavelengths”, NPL Report DES 95, September 1989.

[22] A R Kerr, E Wollack, and N Horner, "Waveguide flanges for ALMA instrumentation,” ALMA Memo. 278, Nov. 1999 [Online]. Available: http://www.alma.nrao.edu/memos/

[23] A R Kerr, "Mismatch caused by waveguide tolerances, corner radii, and flange misalignment," Electronics Div. Tech. Note 215, Jan. 2010 [Online]. Available: http://www.gb.nrao.edu/electronics/edtn/edtn215.pdf

[24] D F Williams, “500 GHz-750 GHz Rectangular-Waveguide Vector-Network-Analyzer Calibrations”, IEEE Transactions on Terahertz Science and Technology, Vol1, No2, pp364-377, April 2011.

[25] H Li et al, "Repeatability of Waveguide Flanges with Worst-Case Tolerances in the 500 - 750 GHz Band", in Microwave Measurement Conference (ARFTG), 2012 79th ARFTG , vol., no., pp.1-8, 22 June 2012.

[26] IEEE P1785.2/D2a, "IEEE Draft Standard for Rectangular Metallic Waveguides and Their Interfaces for Frequencies of $110 \mathrm{GHz}$ and Above-Part 2: Waveguide Interfaces”, 2015. 